\title{
Hollow-core NANF for High-speed Short-reach Transmission in the $\mathrm{S}+\mathrm{C}+\mathrm{L}-$ bands
}

\author{
Yang Hong, Member, IEEE, Thomas D. Bradley, Natsupa Taengnoi, Kyle R. H. Bottrill, \\ John R. Hayes, Gregory T. Jasion, Francesco Poletti, Periklis Petropoulos, Fellow, OSA and \\ David J. Richardson, Fellow, IEEE
}

\begin{abstract}
Hollow-core fibers offer a range of beneficial properties for high-speed optical communication applications, including low latency, low chromatic dispersion and nonlinearity. Based on a system employing a $\sim 5-\mathrm{km}$ span of hollow-core nested antiresonant nodeless fiber (NANF), we demonstrate in this paper, penalty-free Nyquist 4-ary pulse amplitude modulation transmission relative to the back-to-back at 80,100 and $112 \mathrm{~Gb} / \mathrm{s}$ over the $\mathrm{S}+\mathrm{C}+\mathrm{L}-$ bands. Furthermore, using multi-carrier directdetection optical orthogonal frequency division multiplexing, we show that compared to a standard single-mode fiber of a similar length, the NANF exhibits a significantly better bit error rate (BER) performance at $1550 \mathrm{~nm}$ and offers more than $20 \%$ capacity enhancement. Consequently, up to $138.09-\mathrm{Gb} / \mathrm{s}$ capacity has been achieved at a BER threshold of $3.8 \times 10^{-3}$ after $\sim 5-\mathrm{km}$ transmission. We conclude that the use of NANF offers a promising solution for achieving simultaneously high capacity and low latency in future short-reach optical interconnects.
\end{abstract}

Index Terms-Hollow-core Fibers; Ultra-wideband Transmission; Intensity-modulation and Direct-detection; Shortreach Optical Interconnects.

\section{INTRODUCTION}

$\mathrm{H}$ OLLOW-CORE optical fibers based on nested antiresonant nodeless fiber (NANF) technology have emerged recently as a promising evolution from conventional standard single mode fiber (SSMF) for optical data transmission [1-2]. NANFs can be designed to offer low-loss and effectively single-mode propagation over an extended optical bandwidth [3-4]. Since they rely on guidance in a hollow core, they benefit from propagation speeds that approach the speed of light in vacuum, thereby offering approximately $30 \%$ lower latency in transmission than SSMFs [2]. Furthermore, hollow-core guidance also results in much more linear optical fiber transmission, thereby avoiding the onset of deleterious nonlinear effects. These benefits offer the potential to revolutionize the design of optical networks once multikilometer long fiber cables exhibiting similar/lower losses than SSMF can be robustly manufactured and deployed. Moreover,

Manuscript was received XX xx, 2021. This work was supported by the UK's EPSRC under the Airguide Photonics Programme grant (EP/P030181/1), the COALESCE project (EP/P003990/1), the Royal Academy of Engineering, and the ERC Lightpipe project (682724). (Corresponding author: Yang Hong).

The authors are with the Optoelectronics Research Centre, University of Southampton, Southampton SO17 1BJ, UK. (e-mails: y.hong@soton.ac.uk; an additional benefit of hollow-core guidance in NANFs is that the fiber chromatic dispersion (CD) takes extremely low values over an extended wavelength region away from the edges of its passband [5]. While the loss of NANFs has been reduced rapidly over the last few years [6-8], the low CD of NANF promises to have more immediate impact in shorter-reach optical systems, which are not loss-limited. For example, the low $\mathrm{CD}$ may alleviate the power fading issue in intensitymodulation/direct-detection (IM/DD) systems and also allow for the use of less complex equalization for CD compensation/mitigation in coherent transmission.

In this context, this paper considers short-reach $(<10 \mathrm{~km}$ long) optical links, which play a vital role in today's optical networks, since they are the dominant contributors to data traffic [9-11]. Of increasing importance are connections between edge data centers, edge nodes and data producers/consumers [12] which support rapidly expanding traffic demand and enable low-latency applications in today's networks. Driven by this, the realization of high-speed shortreach optical interconnects has drawn significant research interest from both academia and industry in recent years [1314]. To date, intensity-modulation/direct-detection (IM/DD) is the dominant solution for short-reach interconnects with link lengths ranging from a few meters to a few kilometers. This is primarily because of the inherent advantages of IM/DD in terms of simplicity, cost, power consumption and footprint over coherent approaches [14-15]. Moving towards the standardization of $400 \mathrm{G}$ systems, IM/DD is still prioritized for such short-reach applications [16-17], and the IEEE has established the P802.3bm Task Force to address this demand [18]. Meanwhile, it is worth noting that the use of coherent detection in short-reach access is also currently being considered, and is included in the 400ZR implementation agreement by the Optical Internetworking Forum [19]. While there have already been some demonstrations that highlight the NANF's properties which would be greatly beneficial to highspeed coherent transmission systems [20-21], in this work, our focus lies in the use of NANF for IM/DD applications.

In the literature, research and development efforts have

t.bradley@soton.ac.uk; nt1a15@soton.ac.uk; $\quad$ k.bottrill@soton.ac.uk; jrh@orc.soton.ac.uk; g.jasion@soton.ac.uk; $\quad$ frap@orc.soton.ac.uk; pp@orc.soton.ac.uk; djr@orc.soton.ac.uk)

Color versions of one or more of the figures in this paper are available online at http://ieeexplore.ieee.org.

Digital Object Identifier: XXXXXX 
tackled different aspects of high-speed IM/DD transmission, including transceivers, advanced digital signal processing (DSP) and modulation formats [22-28]. With respect to the latter, 4-ary pulse amplitude modulation (PAM4), carrierless amplitude and phase (CAP) modulation, and DD optical orthogonal frequency division multiplexing (DDO-OFDM) with adaptive bit-and-power loading (also known as discrete multitone (DMT) modulation), have been extensively investigated [27-28]. An experimental comparison amongst different modulation formats in $100-\mathrm{Gb} / \mathrm{s} / \lambda$ short-reach interconnects was reported in [28], showing their respective advantages in terms of receiver sensitivity, spectral efficiency, and low computational complexity. However, it is challenging to realize high-speed C-band transmission over SSMF with IM/DD technology, an important performance-limiting factor being CD-induced power fading [29]. For example, it is straightforward to show that the spectrum of broadband signals will experience a first spectral null at $\sim 25 \mathrm{GHz}$ after transmission over 5-km of SSMF at $1550 \mathrm{~nm}$. Generally, CD effects in short-reach SSMF links are mitigated either by using spectrally-efficient modulation formats in C-band transmission systems [28-29] or by adopting the O-band for data transmission. However, the O-band is highly susceptible to nonlinearities of SSMFs even for short-reach links [30] due to the low $\mathrm{CD}$ in this spectral region, which restricts the number of high-speed wavelength-division multiplexed (WDM) channels. In contrast, hollow-core NANFs are effectively nonlinearity-free [21] and exhibit ultra-low $\mathrm{CD}$ over a broad bandwidth, thus offering the potential to achieve extremely high capacities using wideband densely-spaced WDM transmission.

In this work, we study IM/DD systems that use either Nyquist PAM4 or adaptively-loaded DDO-OFDM, and are constructed based on a $\sim 5-\mathrm{km}$ NANF span. We demonstrate that relative to the back-to-back (B2B), NANF allows for penalty-free Nyquist PAM4 transmission over the $\mathrm{S}+\mathrm{C}+\mathrm{L}-$ bands at speeds of 80,100

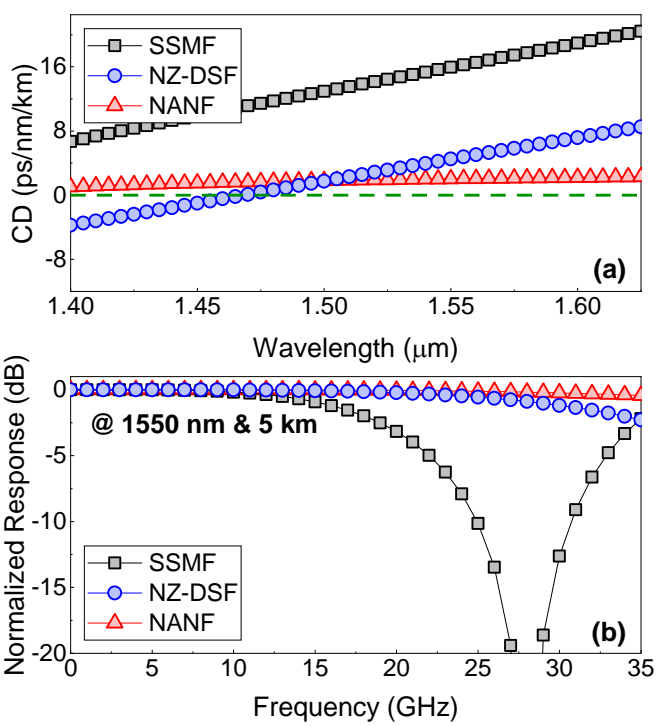

and $112 \mathrm{~Gb} / \mathrm{s}$ after transmission in $\sim 5 \mathrm{~km}$ of this fiber. This performance significantly outperforms that of a similar length of SSMF. Using the adaptively-loaded DDO-OFDM, we further show that $>20 \%$ capacity improvement over a SSMF of a similar length can be achieved in NANF, and up to 138.09$\mathrm{Gb} / \mathrm{s} \mathrm{IM} / \mathrm{DD}$ transmission can be realized at a bit error rate (BER) threshold of $3.8 \times 10^{-3}$.

The rest of this paper is organized as follows. Section II presents a numerical comparison between SSMF and NANF. Section III shows the detailed experimental setup used in our transmission experiments. The results of Nyquist PAM4 transmission are given in Section IV whilst the results of DDOOFDM are shown in Section V. Finally, Section VI concludes this work. This paper expands on the results that were originally presented in [5].

\section{NUMERICAL SimULATIONS}

Fig. 1 shows the results of a numerical comparison between SSMF (ITU G.652 [31]) and a NANF (whose design has been detailed in [32]) of 5-km length. For reference, we also show the results obtained for a non-zero dispersion-shifted fiber (NZDSF, ITU G.655 [33]), as an additional low-CD solid-core fiber alternative for the $\mathrm{C}$-band. We first compared the simulated $\mathrm{CD}$ of the fibers over the S+C+L-bands. The SSMF and NZ-DSF calculations assumed the mean values of the $\mathrm{CD}$ range, as specified in the ITU standards for the wavelengths of interest [31,33]. It is seen in Fig. 1(a) that the NANF has a much lower $\mathrm{CD}$ as well as a much lower CD slope than SSMF across the 1.4 to $1.625-\mu \mathrm{m}$ wavelength range. It is worth noting that the $\mathrm{CD}$ is directly responsible for power fading in IM/DD, which imposes a limit on the usable bandwidth at a given wavelength and transmission distance. An example of this is shown in Fig. 1(b), which compares the frequency response of the three fiber types at a wavelength of $1550 \mathrm{~nm}$ and over a distance of $5 \mathrm{~km}$. The CD values of the SSMF, NZ-DSF and NANF at this wavelength
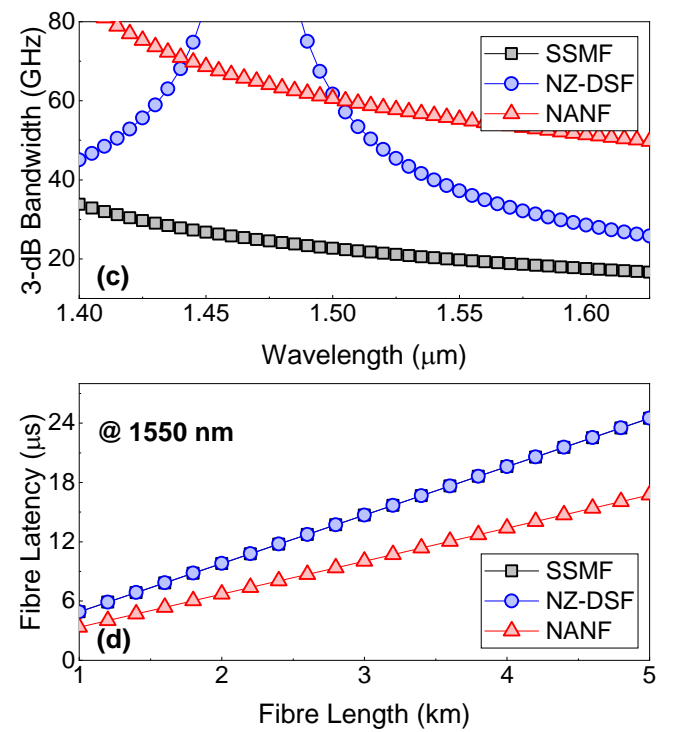

Fig. 1. Numerical comparisons amongst the SSMF, NZ-DSF and NANF at a length of $5 \mathrm{~km}$ : (a) CD, (b) 3-dB bandwidth, (c) profiles of CD-induced power fading after 5-km 1550-nm transmission and (d) fibre latency at $1550 \mathrm{~nm}$. Note that the results of SSMF and NZ-DSF overlapped with each other in Fig. 1(d). 
have been assumed to be $15.95,4.50$ and $2.04 \mathrm{ps} / \mathrm{nm} / \mathrm{km}$, respectively. The $3-\mathrm{dB}$ bandwidth of the three fiber types is compared in Fig. 1(c) across the 1.4 to $1.625-\mu \mathrm{m}$ wavelength range. It is observed that the NANF exhibits more than twice the bandwidth across this entire wavelength range compared to the SSMF. It also offers a wider bandwidth than the NZ-DSF, apart from within a $\sim 60-\mathrm{nm}$ range centered around $1460 \mathrm{~nm}$, where the CD of the NZ-DSF approaches zero. We note that although the bandwidth of the NZ-DSF is broader than that of the NANF in this $\sim 60 \mathrm{~nm}$ window, the available bandwidth in the NANF $(>60 \mathrm{GHz})$ is already well above the bandwidth requirements of 400/800G standards [16-18]. Furthermore, this graph does not account for the effects of fiber nonlinearities, which are more pronounced in the low-CD region of the NZDSF (i.e. where its bandwidth is the broadest), and which in turn will restrict the fiber's capability for wavelength-division multiplexing transmission [34]. In contrast, the NANF is effectively free of nonlinearities. We also note that discussion of fiber loss was excluded from the numerical simulations when evaluating the $\mathrm{CD}$ effect in the three types of fibers. The impact of fiber loss on transmission, which is also an important parameter in this work, will be experimentally evaluated in Section IV. We further investigated the latency performance of the different fibers, using (effective) refractive indices of 1.4682 (Corning SMF28e), 1.4693 (Corning LEAF), and 1.003 [2] for the SSMF, NZ-DSF and NANF, respectively. The results are shown in Fig. 1(d). It is seen that even at a link length of just $5 \mathrm{~km}$, the NANF offers a $\sim 8$ - $\mu$ s latency reduction compared to the two solid-core fibers.

\section{EXPERIMENTAL SETUP}

\section{A. System configuration}

To evaluate the transmission performance in SSMF and NANF, we implemented an IM/DD transmission system, as shown in Fig. 2 (note that the case of NZ-DSF was not considered, since a suitable length of NZ-DSF was not available while performing these experiments). At the transmitter, a tunable laser capable of generating an optical carrier over the range from $1460 \mathrm{~nm}$ to $1640 \mathrm{~nm}$ was connected to a MachZehnder modulator (MZM), which was driven by an electrically amplified data signal generated by an arbitrary waveform generator (AWG) followed by an electrical amplifier (EA). The output of the MZM was directly launched into the transmission link (either NANF or SSMF) without optical amplification. In addition, we have also considered the $\mathrm{B} 2 \mathrm{~B}$ case, as indicated in Fig. 2. In the NANF case, two structurally matched NANFs were directly spliced together to produce a spool of $\sim 4.76 \mathrm{~km}$ length. We note that similar to the splicing of SSMFs, the NANFs were arc-fusion spliced after having been structurally aligned with each other, and the splice loss was around $0.1 \mathrm{~dB}$. The average core size and membrane thickness of the first $~ 3.4-$ $\mathrm{km}$ length of NANF (\#1) were $36.4 \mu \mathrm{m}$ and $0.55 \mu \mathrm{m}$, respectively, whilst the corresponding values of the second $\sim 1.4-\mathrm{km}$ length of NANF (\#2) were $34.6 \mu \mathrm{m}$ and $0.50 \mu \mathrm{m}$, respectively [32]. Scanning electron microscope (SEM) images of the two NANFs are shown in Fig. 2, showing symmetric fiber cross-sections. The two ends of the spliced NANF were connectorized through SSMF fiber pigtails, and these multiple splices contributed to around $2 \mathrm{~dB}$ loss. The total loss of the SSMF-connectorized NANF ranged from 6.45 to $8.33 \mathrm{~dB}$ over the $\mathrm{S}+\mathrm{C}+\mathrm{L}-$ bands, as will be detailed in Section IV below. In the SSMF case, the total fiber length was $\sim 4.53 \mathrm{~km}$, which comprised two spools of $\sim 4-\mathrm{km}$ and $\sim 0.5 \mathrm{~km}$ lengths, respectively.

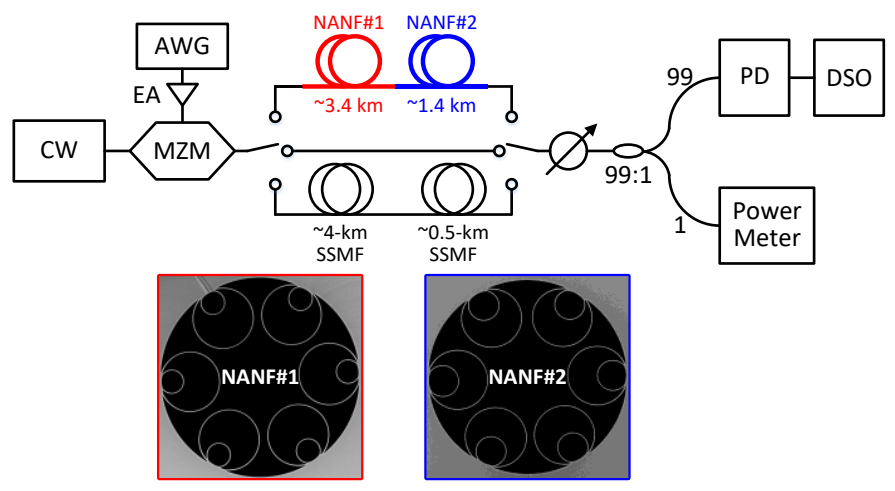

Fig. 2. Experimental setup of the IM/DD system. Insets: SEM images of the used NANFs.

At the receiver, an optical attenuator was used to vary the received optical power (ROP) at the photodetector (PD), which was monitored using a 99:1 optical coupler. The detected electrical signal at the PD was then recorded by an $80-\mathrm{GSa} / \mathrm{s}$ digital storage oscilloscope (DSO) for further offline DSP.

\section{B. DSP blocks}

Different DSP processes were required for the aforementioned two modulation formats in this work. For the generation of the single-carrier Nyquist PAM4 (Fig. 3(a)), the PAM4-mapped signal was first 70 times up-sampled and then Nyquist-filtered using a square-root raised cosine (SRRC) filter. The roll-off factor of the SRRC filter was 0.1. Then, the filtered signal was 40-/50-/56-times down-sampled and digitalto-analog converted in the AWG operating at $70 \mathrm{GSa} / \mathrm{s}$, to generate $80-/ 100-/ 112-\mathrm{Gb} / \mathrm{s}$ Nyquist PAM4 signals, respectively. At the receiver side, the synchronized data was resampled and match-filtered before being fed to a $T / 2$-spaced $(17,7)$-tap decision feedback equalizer (DFE). The recursive least squares (RLS) algorithm was used in the DFE and its forgetting factor was 0.998 . Finally, the equalized signal was PAM4 de-mapped and the corresponding BER was calculated via error counting.

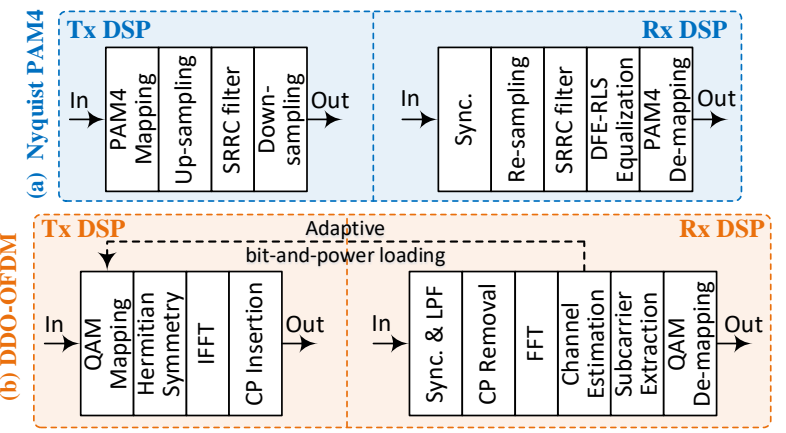

Fig. 3. Block diagrams of (a) the Nyquist PAM4 and (b) the DDO-OFDM. 

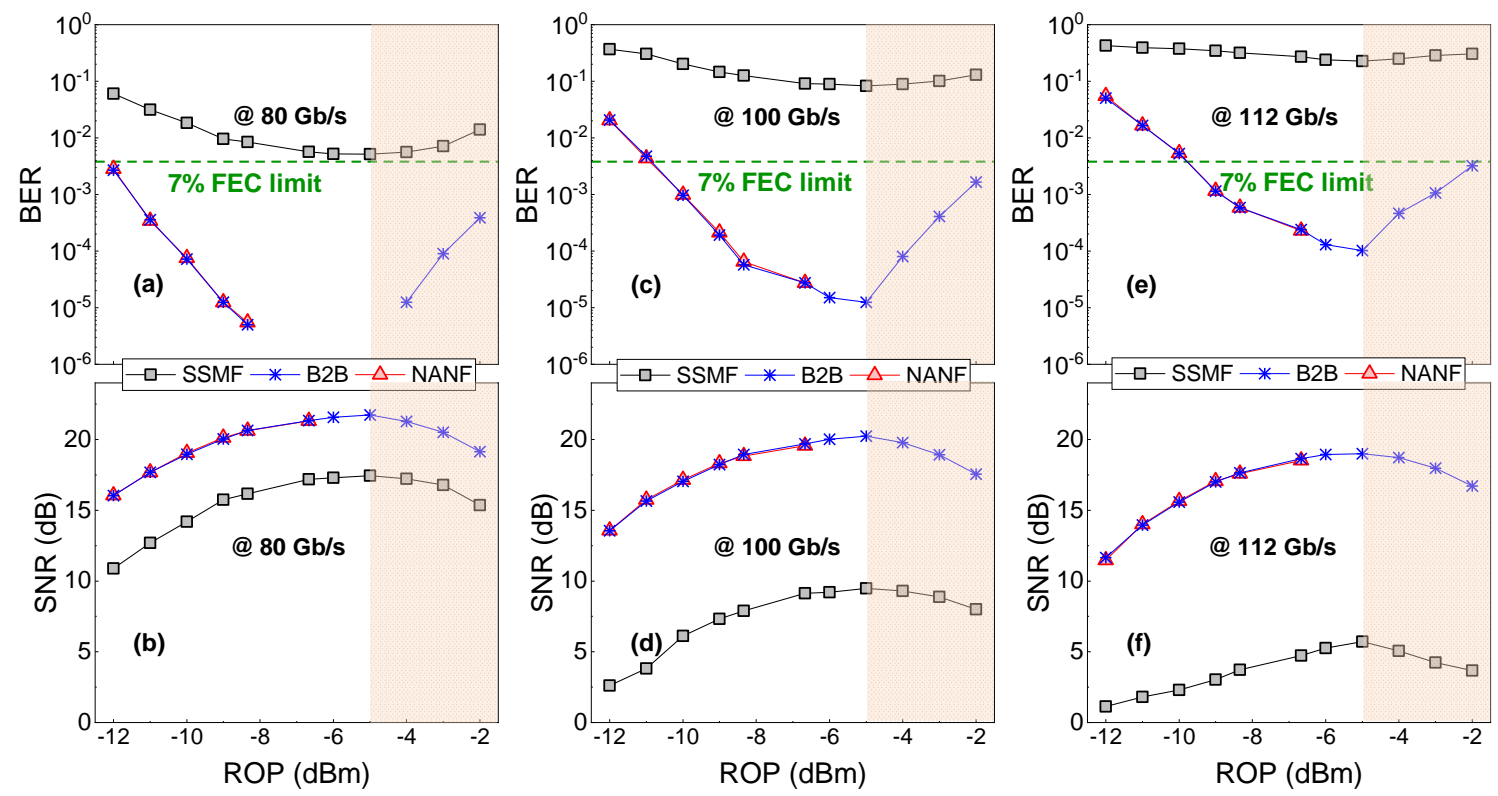

Fig. 4. Comparisons of BER and SNR performance versus ROP of 1550-nm Nyquist PAM4 transmission in SSMF, B2B and NANF links at (a)-(b): 80 Gb/s, (c)(d): $100 \mathrm{~Gb} / \mathrm{s}$, and (e)-(f): $112 \mathrm{~Gb} / \mathrm{s}$. Shaded region: the limited power range of the transimpedance amplifier causes spurious results when ROP > $-5 \mathrm{dBm}$.

For the DDO-OFDM transmitter (Fig. 3(b)), after quadrature amplitude modulation (QAM) mapping, Hermitian symmetry operation was implemented to ensure real-valued outputs after the 512-point inverse fast Fourier transform (IFFT). Before being sent to the 70-GSa/s AWG, a cyclic prefix with a length of 1/16 DDO-OFDM symbols was adopted. At the receiver, the recorded data was synchronized and low-pass filtered, followed by the CP removal. Then, simple one-tap equalization was performed, and only the data subcarriers were extracted for the subsequent QAM de-mapping. The corresponding BER was also obtained via error counting. For the adaptively-loaded DDO-OFDM, pilot transmission was conducted to estimate the channel state information, which was then utilized at the transmitter for adaptive bit-and-power loading [4, 35].

\section{SINGLE-CARRIER NYQUIST-PAM4 TRANSMISSION}

In this section, we investigate the transmission performance of the Nyquist PAM4 signals in the B2B, SSMF and NANF links. To characterize the impact of $\mathrm{CD}$ on high-speed transmission, the data rates of 80,100 and $112 \mathrm{~Gb} / \mathrm{s}$ were considered in the experiments.

\section{A. BER\&SNR versus ROP at $1550 \mathrm{~nm}$}

We first studied the BER and signal-to-noise ratio (SNR) performance versus ROP at different speeds for the three links at the wavelength of $1550 \mathrm{~nm}$. Fig. 4 shows the corresponding transmission results. It is seen that under all the investigated data rates (i.e., 80, 100 and $112 \mathrm{~Gb} / \mathrm{s}$ ), similar BERs and SNRs as for the B2B were achieved in the NANF, confirming that the fiber maintains the integrity of the signals over this distance. In both of these cases, the required ROPs to obtain a BER below $3.8 \times 10^{-3}$, i.e., the $7 \%$ forward error correction (FEC) limit, were around $-12 \mathrm{dBm},-11 \mathrm{dBm}$, and $-10 \mathrm{dBm}$, when transmitting at $80-\mathrm{Gb} / \mathrm{s}, 100-\mathrm{Gb} / \mathrm{s}$ and $112-\mathrm{Gb} / \mathrm{s}$, respectively. In contrast, regardless of the ROP, the BER measured when transmitting in the SSMF was always above the 7\% FEC limit, even for the case of $80-\mathrm{Gb} / \mathrm{s}$. Indeed, an $80-\mathrm{Gb} / \mathrm{s}$ PAM4 signal has a $3-\mathrm{dB}$ bandwidth of $\sim 20 \mathrm{GHz}$, and Fig. 1(b) suggests that it will be shaped substantially due to power fading in the dispersive fiber. This effect is also reflected in the SNR plots of Figs. 4(b), 4(d) and 4(f), which confirm that transmission at $80-\mathrm{Gb} / \mathrm{s}, 100-\mathrm{Gb} / \mathrm{s}$ and $112-\mathrm{Gb} / \mathrm{s}$ over the SSMF suffers an additional penalty of around $5 \mathrm{~dB}, 11 \mathrm{~dB}$ and $14 \mathrm{~dB}$, respectively, relative to the NANF (and B2B).

It is noted that when increasing the ROP to values beyond $-5 \mathrm{dBm}$, degraded BER and SNR performance was experienced in all cases, as shown in the shaded region in Fig. 4. This was because of the limited input power range of the transimpedance amplifier in the PD. Therefore, in the following experiments, to avoid the high-ROP-induced performance degradation, an upper ROP limit of $-5 \mathrm{dBm}$ was imposed on the PD.

\section{B. BER\&SNR performance over $S+C+L$-bands}

We next tuned the laser across the $\mathrm{S}+\mathrm{C}+\mathrm{L}-$ bands to investigate the transmission performance in $\mathrm{B} 2 \mathrm{~B} / \mathrm{SSMF} / \mathrm{NANF}$ links. While doing this, the total loss of the $\sim 5-\mathrm{km}$ SSMFconnectorized NANF over the $\mathrm{S}+\mathrm{C}+\mathrm{L}$-bands was experimentally characterized, and the corresponding results are shown in Fig. 5. 

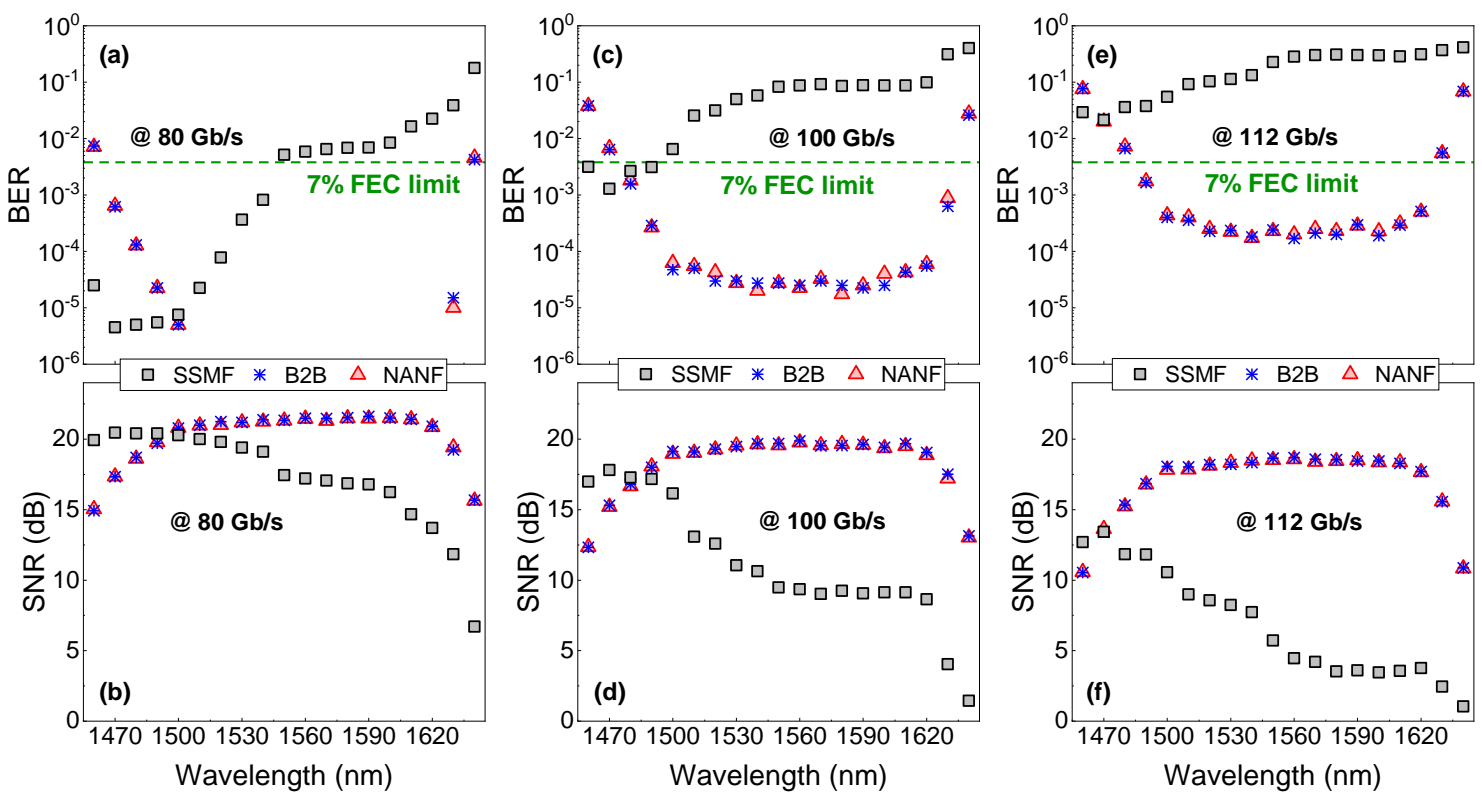

Fig. 6. Comparisons of BER and SNR performance across the S+C+L-bands of Nyquist PAM4 transmission in SSMF, B2B and NANF links at (a)-(b): 80 Gb/s, (c)-(d): $100 \mathrm{~Gb} / \mathrm{s}$, and (e)-(f): $112 \mathrm{~Gb} / \mathrm{s}$.

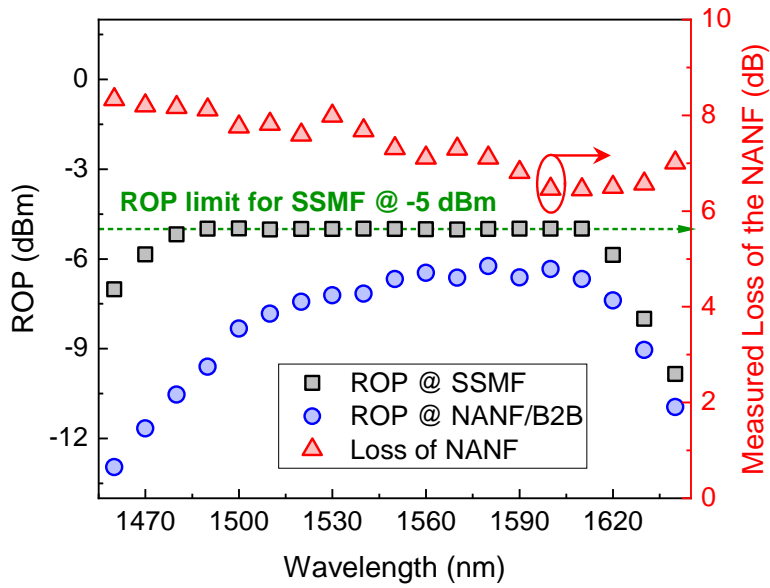

Fig. 5. ROP in different links and the measured loss of the SSMF-connectorized NANF over S+C+L-bands.

The figure shows that the insertion loss of the NANF link ranged from 6.45 to $8.33 \mathrm{~dB}$ in the spectral region of interest. Note that these values include the aforementioned $\sim 2-\mathrm{dB}$ splicing losses. A minimum loss of $6.45 \mathrm{~dB}$ was measured at $1610 \mathrm{~nm}$.

The transmission performance at the three data rates was then measured across this entire wavelength range. This was evaluated at the maximum ROP that was available, and which is also plotted in Fig.5. Note that in order to ensure a meaningful comparison with the B2B case, the ROPs for the B2B link were maintained at the same values as in the NANF case. In the case of the SSMF, the ROP was capped at $-5 \mathrm{dBm}$, to avoid nonlinear distortions at the PD, as mentioned above. The reduced ROPs at the two edges of the spectral region were due to the limited power provided by our tunable laser at these wavelengths.

The results of the $80-\mathrm{Gb} / \mathrm{s}, 100-\mathrm{Gb} / \mathrm{s}$ and $112-\mathrm{Gb} / \mathrm{s}$ Nyquist PAM4 transmission across the $\mathrm{S}+\mathrm{C}+\mathrm{L}-\mathrm{b} a n d s$ are shown in Fig. 6. It is evident that similar BER and SNR values can be achieved over the investigated spectral region at all these data rates for the NANF as in the B2B, validating the capability of the NANF for penalty-free high-speed transmission over an ultra-wide bandwidth after around $5 \mathrm{~km}$. Across all C-band wavelengths and most of the S- and L-band wavelengths, a BER lower than the 7\% FEC limit can be achieved in the NANF even at the highest data rate of $112 \mathrm{~Gb} / \mathrm{s}$ that we considered. In contrast, only a few wavelengths at the short-wavelength edge of the S-band can marginally achieve a BER below $3.8 \times 10^{-3}$ in the SSMF at data rates above $100 \mathrm{~Gb} / \mathrm{s}$. At the lower data rate of $80 \mathrm{~Gb} / \mathrm{s}$, acceptable BER performance $\left(<3.8 \times 10^{-3}\right)$ was achieved at wavelengths shorter than $1550 \mathrm{~nm}$, where the impact of CD was less severe. Note that it is only at the shorter wavelengths of the S-band, where the higher ROP that was available in the SSMF transmission offered an edge in the performance relative to the NANF, especially for the $80-\mathrm{Gb} / \mathrm{s}$ case (Figs. 6(a) and 6(b)). This resulted both from the higher loss of the NANF, and the diminishing CD values in the SSMF at these wavelengths.

It is also worth noting that the signal performance was largely dependent on the wavelength while transmitting in SSMF (reflecting the substantial changes in the $\mathrm{CD}$ values across this wide spectral range), which is in contrast to the transmission in the NANF, which exhibits almost uniform and low $\mathrm{CD}$ values across this entire spectral range (see Fig. 1(a)).

\section{MULTI-CARRIER DDO-OFDM TRANSMISSION}

We next adopted multi-carrier DDO-OFDM as the modulation format, since its signal bandwidth can be flexibly adjusted by altering the number of data subcarriers, transmission capacity can also be maximized via adaptive bitand-power loading. Since the previous Section ascertained that transmission in the NANF over the $\mathrm{S}+\mathrm{C}+\mathrm{L}$-bands after $\sim 5 \mathrm{~km}$ is penalty-free relative to the $\mathrm{B} 2 \mathrm{~B}$ link, we carried out measurements in this Section at one wavelength only, namely 
$1550 \mathrm{~nm}$. We started by comparing the performance of uniformly-loaded DDO-OFDM in the NANF and the SSMF, and then studied the transmission of adaptively-loaded DDOOFDM in the two fiber types.

\section{A. Performance of the uniformly-loaded DDO-OFDM}

Fig. 7 shows a comparison of the BER and average SNR performance of uniformly-loaded DDO-OFDM transmission in SSMF and NANF, for a varying number of subcarriers and a fixed ROP of $-7 \mathrm{dBm}$. Here, the average SNR refers to the mean value of the SNRs of all data subcarriers. All the data subcarriers were loaded with 16QAM signals and no power loading was implemented. Therefore, when changing the number of data subcarriers from 100 to 240 , the corresponding data rate varied from $54.6875 \mathrm{~Gb} / \mathrm{s}$ to $131.25 \mathrm{~Gb} / \mathrm{s}$. Note that the maximum number of data subcarriers, namely 240 , was limited by the cut-off frequency of the DSO. It is seen that the NANF offered a lower BER and a higher average SNR than the SSMF in all cases, yet the differences in the performance were less pronounced when the number of data subcarriers (hence, the signal bandwidth) was small. In accordance to the discussions in the preceding Sections, this is because the extent of the CD-induced power fading depends on the signal bandwidth.

It is worth noting that with an increase in the number of data subcarriers, i.e. the signal bandwidth, the BER and SNR performance of the NANF transmission also degraded, as shown in Fig. 7. This is attributed to the roll-off effect of the bandwidth-limited transceiver, rather than the NANF itself.

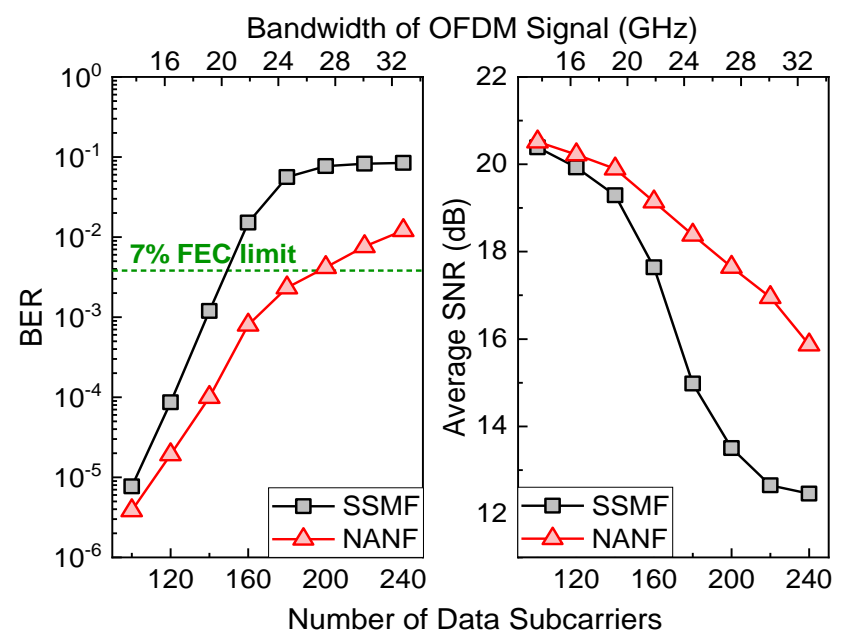

Fig. 7. BER and average SNR versus number of data subcarriers for uniformlyloaded DDO-OFDM transmission in $\sim 5$-km SSMF and NANF. The ROPs in all cases were fixed to $-7 \mathrm{dBm}$.

The SNR profiles of the uniformly-loaded 16QAM-DDOOFDM with 240 data subcarriers after transmission in the NANF and the SSMF are shown in Fig. 8. It is clear that the transmission in the NANF did not suffer from power fading, whereas in the case of the SSMF, a severe spectral null could be observed around $24 \mathrm{GHz}$. Note that the two low-SNR spikes shown in both Figs. 8(a) and 8(b) were caused by clock leakage of the high-speed AWG rather than any of the fibers.

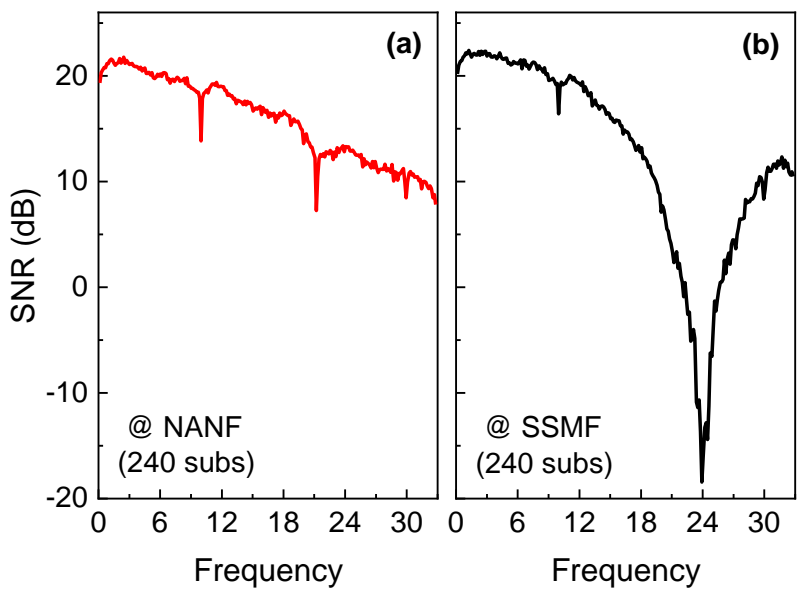

Fig. 8. SNR profiles of uniformly-loaded DDO-OFDM after $\sim 5-\mathrm{km}$ transmission in (a) NANF and (b) SSMF. The ROP in both cases was $-7 \mathrm{dBm}$.

Using 240 data subcarriers in the case of uniformly-loaded DDO-OFDM, i.e. a signal bandwidth of $32.8125 \mathrm{GHz}$, we further varied the ROP at the PD and compared the corresponding performance in the NANF and the SSMF. To obtain acceptable BER performance, all data subcarriers were loaded with 4 QAM signals, hence the data rate was reduced relative to the experiments of Fig.7 to $65.625 \mathrm{~Gb} / \mathrm{s}$. The corresponding transmission results are shown in Fig. 9. Due to the severe spectral fading within the signal bandwidth, the BERs of the SSMF transmission were always above the 7\% FEC limit, regardless of the ROP. In comparison, significant BER performance improvement can be enabled when transmitting the same signal in the NANF. A BER lower than the 7\% FEC limit was achieved for a ROP greater than -10 $\mathrm{dBm}$. Accordingly, the NANF exhibited around 4.5-dB improvement over the SSMF in terms of the average SNR performance.
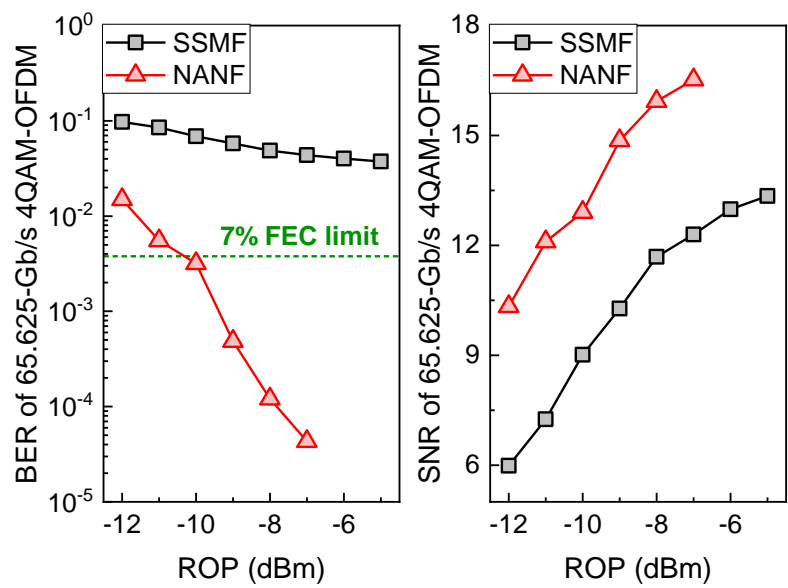

Fig. 9. BER and average SNR versus ROP for $65.625-\mathrm{Gb} / \mathrm{s}$ uniformly-loaded 4QAM-DDO-OFDM transmission in $\sim 5$-km SSMF and NANF.

\section{B. Performance of the adaptively-loaded DDO-OFDM}

The final set of transmission tests used adaptively-loaded DDO-OFDM, validating that the NANF can offer significant performance improvement even when the adaptive bit-andpower loading is employed. 


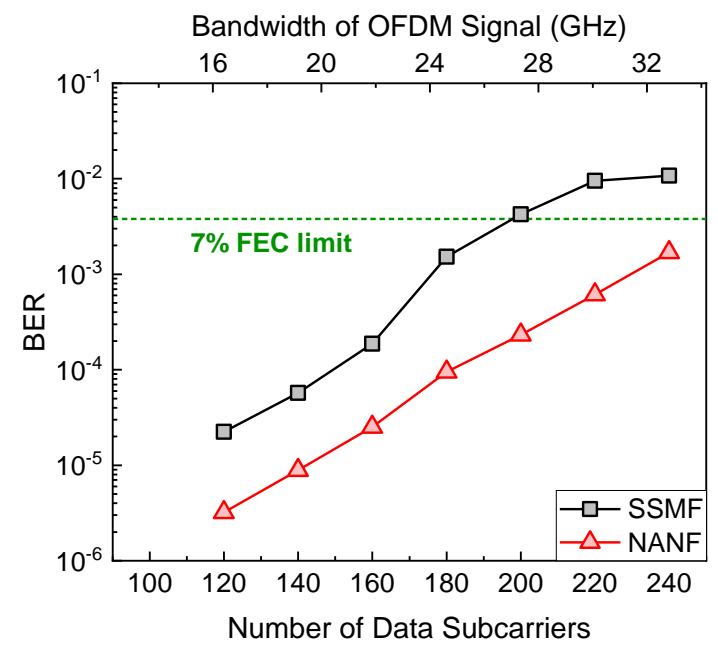

Fig. 10. BER versus number of data subcarriers for adaptively-loaded DDOOFDM transmission in $\sim 5-\mathrm{km}$ SSMF and NANF. The ROPs in all cases were fixed to $-7 \mathrm{dBm}$.

Fig. 10 shows the BER performance of the adaptively-loaded DDO-OFDM transmission as a function of the number of data subcarriers, for a fixed ROP of $-7 \mathrm{dBm}$. To show a fair comparison with the results shown in Fig. 7, the target of the adaptive bit-and-power loading applied here was to optimize the BER performance whilst maintaining the same data rates as those in Fig. 7, i.e. keeping an identical spectral efficiency of $4 \mathrm{bit} / \mathrm{symbol} / \mathrm{Hz}$. Furthermore, since some subcarriers may not be loaded with data, the comparison of average SNR performance is omitted. As anticipated, the figure shows that the BER performance of both SSMF and NANF transmission improved through the use of adaptive bit-and-power loading. As a result, more than 240 subcarriers, i.e. $>131.25 \mathrm{~Gb} / \mathrm{s}$ total capacity, could be supported in the NANF while maintaining a BER below the 7\% FEC limit. The maximum capacity of the SSMF transmission system was $\sim 109.375 \mathrm{~Gb} / \mathrm{s} \quad(\sim 200$ subcarriers) under the same BER threshold.

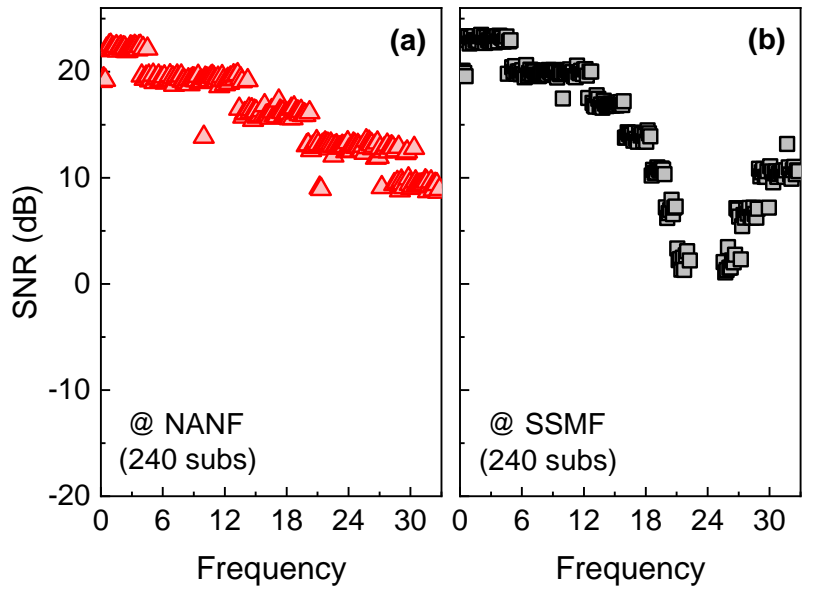

Fig. 11. SNR profiles of adaptively-loaded DDO-OFDM after $\sim 5-\mathrm{km}$ transmission in (a) NANF and (b) SSMF. The ROP in both cases was $-7 \mathrm{dBm}$.

The corresponding SNR profiles of the 240-subcarrier adaptively-loaded DDO-OFDM transmission in the NANF and the SSMF are shown in Fig. 11. Clearly, the profiles generally follow the patterns shown in Fig. 8. The difference is that the subcarriers loaded with the same order of QAM formats shared comparable SNRs and those under extremely low SNR conditions were not data-loaded. This is the case for the subcarriers falling around the spectral null region of the SSMF. In comparison, the SNRs of the subcarriers in the NANF case exhibited a staircase-like profile, since the transmission did not suffer from severe power fading thanks to the ultra-low CD of the fiber.

Finally, we investigated the maximum achievable capacity of the adaptively-loaded DDO-OFDM transmission in the two fiber types under different ROPs, where the objective of the adaptive bit-and-power loading was to maximize the transmission capacity while satisfying the 7\% FEC limit. A signal bandwidth of $32.8125 \mathrm{GHz}$, i.e., 240 data subcarriers, was adopted, and the corresponding results are shown in Fig. 12. At all ROPs, around $20 \%$ to $30 \%$ capacity improvement could be achieved by using the NANF in the transmission relative to the SSMF. After $\sim 5-\mathrm{km}$ transmission, at a ROP of $7 \mathrm{dBm}$, the SSMF enabled a $\sim 112.52-\mathrm{Gb} / \mathrm{s}$ capacity. In comparison, under the same conditions, the NANF could support up to $\sim 138.09 \mathrm{~Gb} / \mathrm{s}$.
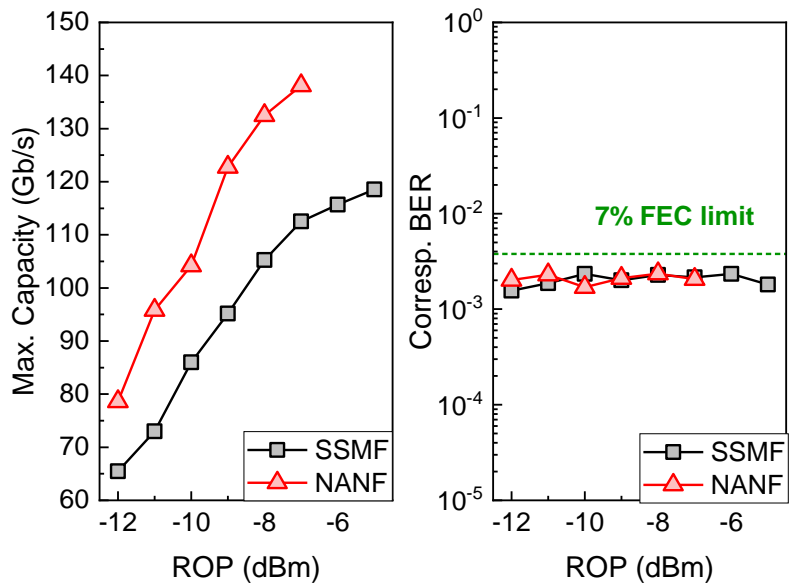

Fig. 12. Comparison of the maximum achievable capacity versus ROP in SSMF and NANF, and the corresponding BER.

\section{CONCLUSION}

In this paper, we have presented a comprehensive experimental study of IM/DD transmission performance in a 5$\mathrm{km}$ length of NANF over the S+C+L-bands. We performed comparative transmission tests over a similar length of SSMF and confirmed that the NANF allows for penalty-free Nyquist PAM4 transmission at speeds of 80,100 and $112 \mathrm{~Gb} / \mathrm{s}$ across this entire wavelength range over a length of $\sim 5 \mathrm{~km}$. This was contrasted to the performance in the SSMF, which was significantly dependent on the signal wavelength and could not support signals with a higher data rate than $80 \mathrm{~Gb} / \mathrm{s}$ for a large proportion of these wavelengths over this distance. We anticipate that the performance gap between the NANF and SSMF in terms of the achievable capacity will further increase with an increase in transmission reach, thanks to the low CD of the NANF. We also adopted the DDO-OFDM format for further performance comparison between the NANF and the SSMF at $1550 \mathrm{~nm}$. We showed that $>20 \%$ capacity enhancement could be achieved in the NANF relative to the 
SSMF at this distance, while operating below the 7\% FEC limit. Consequently, a capacity up to $138.09-\mathrm{Gb} / \mathrm{s}$ was achieved in the $\mathrm{NANF}$ at a ROP of $-7 \mathrm{dBm}$ for the adaptively-loaded DDOOFDM transmission. Our results indicate that even though at their current stage of development, NANFs exhibit a higher propagation loss than SSMF, they already offer significantly better performance in terms of both achievable capacity and latency ( $\sim 30 \%$ reduction) in short-reach IM-DD transmission systems, highlighting their potential for capacity-hungry and latency-sensitive applications like intra-data center links. We believe that the results presented in this work demonstrate a viable route towards fulfilling the increasing capacity and latency requirements of future optical transmission and mobile networks through the adoption of this novel fiber technology.

\section{ACKNOWLEDGMENT}

The data for this work is accessible through the University of Southampton Institutional Research Repository (DOI: https://doi.org/10.5258/SOTON/ D1889).

\section{REFERENCES}

[1] D.J. Richardson, N.V. Wheeler, Y. Chen, et al., "Hollow Core Fibres and their Applications," in Proc. of OFC, Los Angeles, USA, paper Tu3H.1, 2017.

[2] F. Poletti, N. V. Wheeler, M. N. Petrovich, et al., "Towards High-capacity Fibre-optic Communications at the Speed of Light in Vacuum," Nat. Photon., vol. 7, pp. 279-284, 2013.

[3] H. Sakr, Y. Hong, T.D. Bradley, et al, "Interband Short Reach Data Transmission in Ultrawide Bandwidth Hollow Core Fiber," J. Light. Technol., vol. 38, no. 1, pp. 159-165, 2020.

[4] Y. Hong, H. Sakr, N. Taengnoi, et al., "Multi-Band Direct-Detection Transmission Over an Ultrawide Bandwidth Hollow-Core NANF," $J$. Light. Technol., vol. 38, no. 10, pp. 2849-2857, 2020.

[5] Y. Hong, T. D. Bradley, N. Taengnoi, et al., "Comparative Investigations between SSMF and Hollow-core NANF for Transmission in the $\mathrm{S}+\mathrm{C}+\mathrm{L}-$ bands," in Proc. of OFC, San Diego, USA, paper W3E.3, 2020.

[6] T. D. Bradley, J. R. Hayes, Y. Chen, et al., "Record Low-Loss $1.3 \mathrm{~dB} / \mathrm{km}$ Data Transmitting Antiresonant Hollow Core Fibre," in Proc. of ECOC, Rome, Italy, paper PDP Th3F.2, 2018.

[7] T. D. Bradley, G. T. Jasion, J. R. Hayes, et al., "Antiresonant Hollow Core Fibre with $0.65 \mathrm{~dB} / \mathrm{km}$ Attenuation across the $\mathrm{C}$ and $\mathrm{L}$ Telecommunication Bands," in Proc. of ECOC, Dublin, Ireland, paper PD3.1, 2019.

[8] G. T. Jasion, T. D. Bradley, K. Harrington, et al., "Hollow Core NANF with $0.28 \mathrm{~dB} / \mathrm{km}$ Attenuation in the C and L Bands," in Proc. of OFC, San Diego, USA, paper Th4B.4, 2020.

[9] J. Cheng, C. Xie, Y. Chen, et al., "Comparison of Coherent and IMDD Transceivers for Intra Datacenter Optical Interconnects," in Proc. of OFC, San Diego, USA, paper W1F.2, 2019.

[10] R. Urata, H. Liu, X. Zhou, et al., "Data center Interconnect and Networking: from Evolution to Holistic Revolution," in Proc. of OFC, Los Angeles, USA, paper W3G.1, 2017.

[11] Cisco Annual Internet Report. [Online]. Available: https://www.cisco. $\mathrm{com} / \mathrm{c} / \mathrm{en} / \mathrm{us} / \mathrm{solutions/collateral/executive-perspectives/annual-internet-}$ report/white-paper-c11-741490.pdf, Accessed on Jun. 25, 2021.

[12] W. Shi, J. Cao, Q. Zhang, et al., "Edge Computing: Vision and Challenges," IEEE Internet Things J., vol. 3, no. 5, pp. 637-646, 2016.

[13] J. Lee, N. Kaneda, T. Pfau, et al., "Serial 103.125-Gb/s Transmission over $1 \mathrm{~km}$ SSMF for Low-Cost, Short-Reach Optical Interconnects," in Proc. of OFC, San Francisco, USA, paper Th5A.5, 2014.

[14] X. Pang, O. Ozolins, L. Zhang, et al., "Beyond 200 Gbps per Lane Intensity Modulation Direct Detection (IM/DD) Transmissions for
Optical Interconnects: Challenges and Recent Developments," in Proc. of OFC, San Diego, USA, paper W4I.7, 2019.

[15] K. Zhong, X. Zhou, J. Huo, et al., "Digital Signal Processing for ShortReach Optical Communications: A Review of Current Technologies and Future Trends," J. Light. Technol., vol. 36, no. 2, pp. 377-400, 2018.

[16] 400G-BD4.2 Multimode Fiber $8 \times 50$ Gbps Technical Specifications. [Online]. Available: https://www.gigalight.com/downloads/standards/ 400G-BiDi-MSA.pdf, Accessed on Jun. 25, 2021.

[17] 100G Lambda Multi-source Agreement Specifications. [Online]. Available: http://100glambda.com/specifications, Accessed on Jun. 07, 2020

[18] IEEE P802.3bm $40 \mathrm{~Gb} / \mathrm{s}$ and $100 \mathrm{~Gb} / \mathrm{s}$ Fiber Optic Task Force Home Page. [Online]. Available: http://www.ieee802.org/3/bm/, Accessed on Jun. 07, 2020.

[19] Implementation Agreement 400ZR. [Online]. Available: https://www.oiforum.com/wp-content/uploads/OIF-400ZR-01.0_reduce d2.pdf, Accessed on Jun. 26, 2021.

[20] A. Nespola, S. Straullu, T. D. Bradley, et al., "Transmission of 61 C-Band Channels Over Record Distance of Hollow-Core-Fiber With L-Band Interferers," J. Light. Technol., vol. 39, no.3, pp. 813-820, 2021.

[21] Z. Liu, B. Karanov, L. Galdino, et al., "Nonlinearity-Free Coherent Transmission in Hollow-Core Antiresonant Fiber," J. Light. Technol., vol. 37, no.3, pp. 909-916, 2019.

[22] S. Kanazawa, H. Yamazaki, Y. Nakanishi, et al., "Transmission of 214Gbit/s 4-PAM Signal using An Ultra-broadband Lumped-electrode EADFB Laser Module," in Proc. of OFC, Anaheim, USA, paper Th5B.3, 2016.

[23] P.D. Heyn, V.I. Kopp, S.A. Srinivasan, et al., "Ultra-dense $16 \times 56 \mathrm{~Gb} / \mathrm{s}$ NRZ GeSi EAM-PD Arrays Coupled to Multicore Fiber for Short-reach 896Gb/s Optical links," in Proc. of OFC, Los Angeles, USA, paper Th1B.7, 2017

[24] L. Sun, J. Du, C. Wang, et al., "Frequency-resolved Adaptive Probabilistic Shaping for DMT-modulated IM-DD Optical Interconnects," Opt. Express, vol. 27, no. 9, pp. 12241-12254, 2019.

[25] K. Matsumoto, Y. Yoshida, A. Maruta, et al., "On the Impact of Tomlinson-Harashima Precoding in Optical PAM Transmissions for Intra-DCN Communication," in Proc. of OFC, Los Angeles, USA, paper Th3D.7, 2017.

[26] N.P. Diamantopoulos, H. Nishi, W. Kobayashi, et al., "On the Complexity Reduction of the Second-Order Volterra Nonlinear Equalizer for IM/DD Systems," J. Light. Technol., vol. 37, no. 4, pp. 1214-1224, 2019.

[27] Y. Kai, M. Nishihara, T. Tanaka, et al., "Experimental Comparison of Pulse Amplitude Modulation (PAM) and Discrete Multi-tone (DMT) for Short-Reach 400-Gbps Data Communication," in Proc. of ECOC, London, UK, paper Th.1.F.3, 2013.

[28] J. Zhang, J. Shi, and J. Yu, "The Best Modulation Format for 100G Shortreach and Metro Networks: DMT, PAM-4, CAP, or Duobinary?," in Proc. SPIE 10560, Metro and Data Center Optical Networks and Short-Reach Links, paper 1056002, 2018.

[29] Q. Hu, M. Chagnon, K. Schuh, et al., "IM/DD Beyond Bandwidth Limitation for Data Center Optical Interconnects," J. Light. Technol., vol. 37, no. 19, pp. 4940-4946, 2019.

[30] L. Chorchos, and J.P. Turkiewicz, "O-Band 8×100G Data Transmission With 240 GHz Channel Spacing," IEEE Photon. Technol. Lett., vol. 23, no. 12, pp. 2288-2291, 2019.

[31] G.652: Characteristics of A Single-mode Optical Fibre and Cable, ITU Standard, 2016.

[32] A. Nespola, S. Straullu, T. D. Bradley, et al., "Record PM-16QAM and PM-QPSK Transmission Distance (125 and $340 \mathrm{~km}$ ) over Hollow-CoreFiber," in Proc. of ECOC, Dublin, Ireland, paper PD1.5, 2019.

[33] G.655: Characteristics of A Non-zero Dispersion-shifted Single-mode Optical Fibre and Cable, ITU Standard, 2009.

[34] R. W. Tkach, A. R. Chraplyvy, F. Forghieri, et al., "Four-photon Mixing and High-speed WDM Systems," J. Light. Technol., vol. 13, no. 5, pp. 841-849, 1995.

[35] P. S. Chow, J. M. Cioffi, and J. A. C. Bingham, "A Practical Discrete Multitone Transceiver Loading Algorithm for Data Transmission over Spectrally Shaped Channels," IEEE Trans. on Commun., vol. 73, no. 2, pp. 773-775, 1995. 